\title{
Protective effect of berberine on renal fibrosis caused by diabetic nephropathy
}

\author{
ZHONG LI and WEI ZHANG

\begin{abstract}
The Clinical Teaching and Research Department, School of Zhang Zhongjing Traditional Chinese
Medicine, Nanyang Institute of Technology, Nanyang, Henan 473004, P.R. China
\end{abstract}

Received March 15, 2016; Accepted March 10, 2017

DOI: $10.3892 / \mathrm{mmr} .2017 .6707$

\begin{abstract}
Berberine (BBR) is a material extracted from Chinese herbs, which has been used in the treatment of diabetes in Chinese medicine for thousands of years. However, the importance of BBR in renal fibrosis remains to be elucidated. In the present study, streptozotocin-induced diabetic nephropathy (DN) rats were used to determine the effect of BBR on renal fibrosis. The pathology of the kidneys was examined using periodic acid-Schiff (PAS) and Masson staining. The expression levels of transforming growth factor- $\beta$ (TGF- $\beta$ ) and $\alpha$ smooth muscle actin ( $\alpha$-SMA) in kidneys were observed using immunohistochemical staining. The mRNA and protein expression levels of TGF- $\beta, \alpha-$ SMA, vimentin, nuclear factor- $\kappa \mathrm{B}$ were examined using reverse transcription-quantitative polymerase chain reaction and western blotting, respectively. PAS and Masson staining revealed that there was notable glomerular hypertrophy and mesangial matrix expansion in DN rats. Immunohistochemistry revealed that there was a significant increase in TGF- $\beta$ and $\alpha$-SMA expression levels in the renal tubulointerstitium and the extracellular matrix. However, treatment with BBR may significantly reduce kidney injury. The protein and mRNA expression levels of TGF- $\beta$, vimentin and $\alpha$-SMA were significantly increased in DN rats compared with the control group; however, this increase was reduced following treatment with BBR. The present study revealed that BBR may inhibit fibrosis and ameliorate the symptoms of $\mathrm{DN}$. The current findings indicated that BBR may be used as a potential treatment for patients with DN.
\end{abstract}

\section{Introduction}

Diabetes and its associated complications, such as diabetic nephropathy (DN) have become a serious health problem.

Correspondence to: Miss Zhong Li, The Clinical Teaching and Research Department, School of Zhang Zhongjing Traditional Chinese Medicine, Nanyang Institute of Technology, 80 Chang Jiang Road, Nanyang, Henan 473004, P.R. China

E-mail: nylizhong@163.com

Key words: diabetic nephropathy, fibrosis, berberine, kidney
Approximately one-third of all diabetic patients suffer from DN (1), which has significant social and economic burdens (2) and may be the leading cause of end-stage renal disease (ESRD). In the United States 200,000 patients receive ESRD care due to diabetic kidney disease, with 50,000 new patients starting dialysis yearly $(3,4)$. The primary indicators of DN are continuous albuminuria, high blood pressure and progressive renal damage. However, the specific pathogenic mechanisms remain to be fully elucidated. Hyperglycemia has an important part in the development of DN; however, additional factors, such as inflammation due to fibrosis, are considered to be important for the initiation and progression of diabetic nephropathy.

The major pathological alterations of DN include mesangial expansion, extracellular matrix (ECM) alterations, tubulointerstitial fibrosis and glomerular sclerosis. Transforming growth factor- $\beta$ (TGF- $\beta$ ) has been identified to be a key regulator of fibrosis in $\mathrm{DN}$ (5). Overexpression of TGF- $\beta$ may promote epithelial-mesenchymal transition (EMT) and renal sclerosis, ultimately leading to organ failure (6). Chen et al (7) demonstrated that suppression of the TGF- $\beta$ /mothers against decapentaplegic signaling pathway may greatly ameliorate streptozotocin (STZ)-induced fibrosis and albumin levels in the urine of rats (7). Therefore, therapeutic agents that may inhibit TGF- $\beta$ and its signaling pathways may also reduce the progress of DN.

At present, despite the wide use of therapeutic approaches focused on managing hyperglycemia and high blood pressure, numerous patients continue to suffer from progressive and severe renal injury. Therefore, it is important to develop novel renal protective drugs for the treatment of $\mathrm{DN}$. Berberine (BBR) is a type of isoquinolone alkaloid that is extracted from the widely used Chinese herb, Rhizoma coptidis. Recent studies have indicated that BBR has multiple pharmacological activities, including hypolipidemic, antioxidant and glucose-lowering, suggesting that it may have clinical potential as an alternative therapeutic drug for diabetic complications $(8,9)$. Liu et al (10) reported that BBR effectively lowered blood glucose and lipid levels through suppression of oxidative stress. However, it remains to be elucidated if BBR exerts its beneficial effects in DN via regulation of TGF- $\beta$. The present study used a STZ-induced DN rat model to investigate the effect of BBR on the activation of TGF- $\beta$ and its associated pathways in DN. 


\section{Materials and methods}

Materials. Male Wistar rats ( $\mathrm{n}=50$; weight, $150 \pm 10 \mathrm{~g}$ ) were obtained from Laboratory Animal Center of Henan Province (Zhengzhou, China). The rats were raised at an ambient temperature of $24 \pm 1^{\circ} \mathrm{C}$ with 12 -h light/dark cycle and $45 \pm 5 \%$ humidity, and free access to a standard chow diet and water for 1 week prior to the experiment. The present study received ethical approval from the Nanyang Institute of Technology (Nanyang, China).

Rats were randomly assigned into control $(n=10)$ and diabetic $(n=40)$ groups. The rats in the diabetic group received a single intraperitoneal injection of STZ $(60 \mathrm{mg} / \mathrm{kg}$, Sigma-Aldrich; Merck Millipore, Darmstadt, Germany). The control group were injected with citrate buffer solution $(0.1 \mathrm{M}$, $\mathrm{pH}$ 4.4). The development of hyperglycemia was confirmed by measuring fasting blood glucose (FBG) at $72 \mathrm{~h}$ following injection. Those with FBG over $11.1 \mathrm{mM} / 1$ were considered diabetic. A total of 30 diabetic rats were selected and randomly divided into DN, BBR and metformin groups ( $\mathrm{n}=10 /$ group).

The control and DN groups were orally treated with double distilled water $(0.5 \mathrm{ml} / 100 \mathrm{~g})$ per day (vehicle) for 12 weeks. The BBR group received $400 \mathrm{mg} / \mathrm{kg}$ BBR (dissolved in sodium carboxymethyl cellulose and mixed by magnetic stirring apparatus for at least half an hour) orally every day, and the metformin group were treated with $500 \mathrm{mg} / \mathrm{kg}$ metformin solution. The control group rats were fed standard rodent chow and the remaining groups were fed high-fat food (78.8\% normal diet, $10 \%$ lard, $10 \%$ yolk powder, $1 \%$ cholesterol and $0.2 \%$ cholate). All rats had free access to water, and the urine was collected at the end of 83 days from the metabolic cages $24 \mathrm{~h}$ prior to the end of the experiment. At the end of the 12th week, all the animals were anesthetized with $10 \%$ chloralhydrate (Lot 30037574; Sinopharm Chemical Reagent Beijing Co., Ltd.) and sacrificed. Kidney samples were dissected and rapidly excised, weighed and frozen in liquid nitrogen and stored at $-80^{\circ} \mathrm{C}$ or fixed in $10 \%$ neutral-buffered formalin.

Quantification of body weight and urinary albumin. All rats were fasted for $24 \mathrm{~h}$; however, water was placed in the metabolic cages and urine collected prior to sacrifice. Urinary protein excretion was determined by the Bradford method (P0006; Beyotime Institute of Biotechnology, Beijing, China).

Quantification of creatinine $(\mathrm{Scr})$ and blood urea nitrogen $(B U N)$. Serum levels of Scr and BUN were quantified using a Hitachi 7080 Chemistry Analyzer (Hitachi, Ltd., Tokyo, Japan).

Histopathological analysis. Kidney tissues were fixed in $10 \%$ phosphate buffered formalin solution and embedded in paraffin. Paraffin sections (2-3 $\mu \mathrm{m})$ were stained with periodic acid-Schiff (PAS) and Masson's trichrome. The degree of glomerulosclerosis, defined as ECM deposition and mesangial expansion, was randomly selected and evaluated at a magnification of $\mathrm{x} 40$ for 20 cortical fields using a previously described scoring system (0-4 grades) (11).

Immunohistochemistry analysis. Immunohistochemistry was performed on paraffin sections $(3-\mu \mathrm{m})$ using a microwave-based antigen retrieval technique as previously described (12). The slides were incubated overnight at $4^{\circ} \mathrm{C}$ in a humidified chamber with anti-TGF- $\beta$ (sc-101574; 1:500) or anti- $\alpha$-smooth muscle actin ( $\alpha$-SMA; sc-53142; 1:500) (both from Santa Cruz Biotechnology, Inc., Dallas, TX, USA) antibodies. The next day, the sections were incubated with a biotinylated goat-anti-rat secondary antibody (115-035-003; 1:10,000; Jackson ImmunoResearch Laboratories, Inc., West Grove, USA) and subsequently in an avidin-horseradish peroxidase solution. Immunostaining was visualized with $0.05 \%$ diaminobenzidine. Sections were examined using an Olympus DP72 microscope (Olympus Corporation, Tokyo, Japan) and imaged with a high-resolution camera at a magnification of $\mathrm{x} 400$. Areas with positive staining were quantified by using ImageJ version 1.6.0_24 (National Institutes of Health, Bethesda, MD, USA; https://imagej.nih.gov/ij/download.html) and expressed as a percentage of the entire glomerulus or selected tubulointerstitial area.

Reverse transcription-quantitative polymerase chain reaction $(R T-q P C R)$. The renal cortex was collected by carefully dissecting the renal pelvis and medullar tissues, and was subsequently frozen at $-80^{\circ} \mathrm{C}$ for analysis of the gene of interest (13). TRIzol ${ }^{\circledast}$ reagent (Invitrogen; Thermo Fisher Scientific, Inc., Waltham, MA, USA) was used to isolate total RNA from kidney tissues following the manufacturer's protocol. A standard reverse transcriptase reaction kit (Takara Biotechnology, Co., Ltd., Dalian, China) was used to synthesize cDNA. qPCR was performed using the UltraSYBR Mixture kit with ROX I (CW2602M; CW Biotech, Beijing, Beijing, China;) on an ABI 7500 Sequence Detection system (Thermo Fisher Scientific, Inc.). The primers used are presented in Table I. Each sample was analyzed in triplicate. The relative mRNA expression levels were calculated using the $2^{-\Delta \Delta C q}$ method (14). The expression of GAPDH was used as the endogenous reference control.

Western blotting. Renal cortices tissues were lysed in radioimmunoprecipitation (RIPA) buffer (Sanbio, Beijing, China). Proteins from kidney tissues and cultured cells were extracted with RIPA lysis buffer, and western blot analysis was performed as previously described (15). Following centrifugation at $15,000 \mathrm{xg}$ for $15 \mathrm{~min}$ at $4^{\circ} \mathrm{C}$, supernatant protein concentration was quantified using a Bradford assay kit (P0006; Beyotime Institute of Biotechnology). With $50 \mu \mathrm{g}$ proteins were separated by $12 \%$ SDS-PAGE and transferred onto a polyvinylidene difluoride membrane, which was then blocked for $1 \mathrm{~h}$ with $5 \%$ non-fat dry milk in PBS containing $0.1 \%$ Tween-20. The membranes were then incubated with the TGF- $\beta$ (sc-101574; 1:1,000), $\alpha$-SMA (sc-53142; 1:1,000), Vimentin (sc-73259; 1:1,000) (all from Santa Cruz Biotechnology, Inc.), NF-кB (48,862; 1:1,000; Cell Signaling Technology, Inc., Danvers, MA, USA) overnight at $4^{\circ} \mathrm{C}$. The membranes were washed with Tris-buffered saline and incubated for $1 \mathrm{~h}$ with secondary antibodies (111-035-003 or 115-035-003; 1:10,000; Jackson ImmunoResearch Laboratories, Inc.). Following washing, protein bands were detected by a chemiluminescence reagent (Applygen Technologies, Inc., Beijing, China).

Statistical analysis. Data were analyzed using SPSS version 17.0 (SPSS, Inc., Chicago, IL, USA) and are presented as the mean \pm standard deviation. Significance between groups 
Table I. Primers used for quantitative polymerase chain reaction.

\begin{tabular}{llll}
\hline Gene & \multicolumn{1}{c}{ Forward $\left(5^{\prime}-3^{\prime}\right)$} & Reverse (5'-3') & Product length (bp) \\
\hline TGF- $\beta$ & GATACGCCTGAGTGGCTGTCT & GGAAGGGTCGGTTCATGTCAT & 202 \\
$\alpha-S M A$ & TGTACCCAGGCATTGCTGACA & TCTGCTGGAAGGTAGATAAG & 150 \\
Vimentin & GAGATCGCCACCTACAGGAG & TCATCGTGGTGCTGAGAATC & 206 \\
NF- $\kappa$ B & TGCGACAGATGGGCTACACAG & TTTGCGGAAGGATGTCTCCAC & 200 \\
GAPDH & GGTTGTCTCCTGTGACTTCAA & TGCTGTAGCCATATTCATTGT & 125 \\
\hline
\end{tabular}

TGF- $\beta$, transforming growth factor- $\beta$; $\alpha$-SMA, $\alpha$ smooth muscle actin; NF- $\kappa \mathrm{B}$, nuclear factor $\kappa \mathrm{B}$.

Table II. Characteristics of rats in the different treatment groups.

\begin{tabular}{lcccc}
\hline Group & Urine albumin & Glucose & Scr $(\mu \mathrm{M} / \mathrm{l})$ & $\mathrm{BUN}(\mathrm{mM} / \mathrm{l})$ \\
\hline Control & $14.92 \pm 4.23$ & $6.1 \pm 0.6$ & $28.9 \pm 5.2$ & $5.24 \pm 0.36$ \\
DN & $110.3 \pm 12.04^{\mathrm{a}}$ & $21.5 \pm 4.3^{\mathrm{a}}$ & $78.4 \pm 8.9^{\mathrm{a}}$ & $9.71 \pm 0.82^{\mathrm{b}}$ \\
DN+BBR & $87.6 \pm 11.42^{\mathrm{c}}$ & $22.9 \pm 3.7$ & $49.6 \pm 11.1^{\mathrm{c}}$ & $7.33 \pm 1.01^{\mathrm{c}}$ \\
DN+Metformin & $94.51 \pm 16.21$ & $19.1 \pm 6.8$ & $53.4 \pm 7.8^{\mathrm{c}}$ & $6.67 \pm 0.99^{\mathrm{c}}$ \\
\hline
\end{tabular}

Data are presented as mean \pm standard deviation. ${ }^{\mathrm{a}} \mathrm{P}<0.01$ and ${ }^{\mathrm{b}} \mathrm{P}<0.05$ vs. control group, ${ }^{\mathrm{c}} \mathrm{P}<0.05$ vs. DN group. DN, diabetic nephropathy; BBR, berberine; Scr, creatinine; BUN, blood urea nitrogen.

was evaluated by one way analysis of variance followed by a Newman-Keuls post hoc test $\mathrm{P}<0.05$ was considered to indicate a statistically significant difference.

\section{Results}

Effects of BBR on body weight, blood glucose, and 24 h urinary protein. At the end of experiments, rats in DN group experienced significant weight loss $(\mathrm{P}<0.05)$ when compared with the control group (data not shown). No significant difference was identified in terms of body weight in the BBR and metformin groups when compared with the control group. Following induction of diabetes, the blood glucose level was increased in the DN, BBR and metformin groups. However, treatment with BBR and metformin did not significantly decrease the blood level when compared with the DN group (Table II).

During the experimental period, diabetic rats had a significantly greater urine protein excretion. However, after 12-week treatment with BBR, the rats had significantly reduced $24 \mathrm{~h}$ urine albumin when compared with the $\mathrm{DN}$ group $(\mathrm{P}<0.05)$. In addition, High fat food plus STZ injection caused a significant increase of Scr and BUN levels in diabetic rats. However, 12 weeks treatment with BBR can reduced the levels of Scr and BUN compared with the DN group $(\mathrm{P}<0.05$; Table II).

PAS and Masson staining. Both PAS and Masson staining demonstrated significant histological alterations in the DN rats. The DN rats had notable glomerular hypertrophy and mesangial matrix expansion compared to the control group (Fig. 1). However, treatment with BBR or metformin significantly ameliorated these pathological alterations $(\mathrm{P}<0.01$; Fig. 1C).
Immunohistochemical staining of TGF- $\beta$ and $\alpha-S M A$. TGF- $\beta$ and $\alpha$-SMA expression levels in renal tissues were observed by immunohistochemical staining (Fig. 2A and B). The expression levels of TGF- $\beta$ and $\alpha$-SMA in the DN group were increased compared with the normal group. However, BBR treatment ameliorated this effect. In addition, metformin also reduced the expression of TGF- $\beta$ and $\alpha$-SMA; however, there no significant difference was identified between the BBR and metformin groups (Fig. 2C).

Reverse transcription-quantitative polymerase chain reaction (RT-qPCR) and western blotting of TGF- $\beta$, vimentin, $N F-\kappa B$ and $\alpha-S M A$. In order to determine the underlying mechanisms by which BBR inhibits renal fibrosis, the mRNA and protein expression levels of TGF- $\beta$, vimentin, nuclear factor- $\kappa \mathrm{B}(\mathrm{NF}-\kappa \mathrm{B})$ and $\alpha-\mathrm{SMA}$ in diabetic kidney tissues were assessed using RT-qPCR and western blotting, respectively. The mRNA and protein expression levels of TGF- $\beta$, vimentin, and $\alpha$-SMA were significantly increased in the DN group compared with the control group (Fig. 3). However, treatment with BBR may significantly reduce the expression level of those genes $(\mathrm{P}<0.05$; Fig. $3 \mathrm{C})$ when compared with the $\mathrm{DN}$ group. Additionally, no significant difference was identified between the mRNA or protein expression levels of $N F-\kappa B$ in the control group compared with the DN group.

\section{Discussion}

DN is characterized by glomerular hypertrophy, thickness of the basement, tubular and glomerular membranes and accumulation of ECM, which leads to tubulointerstitial and glomerular fibrosis and sclerosis. TGF- $\beta$ has a key role in the 
A
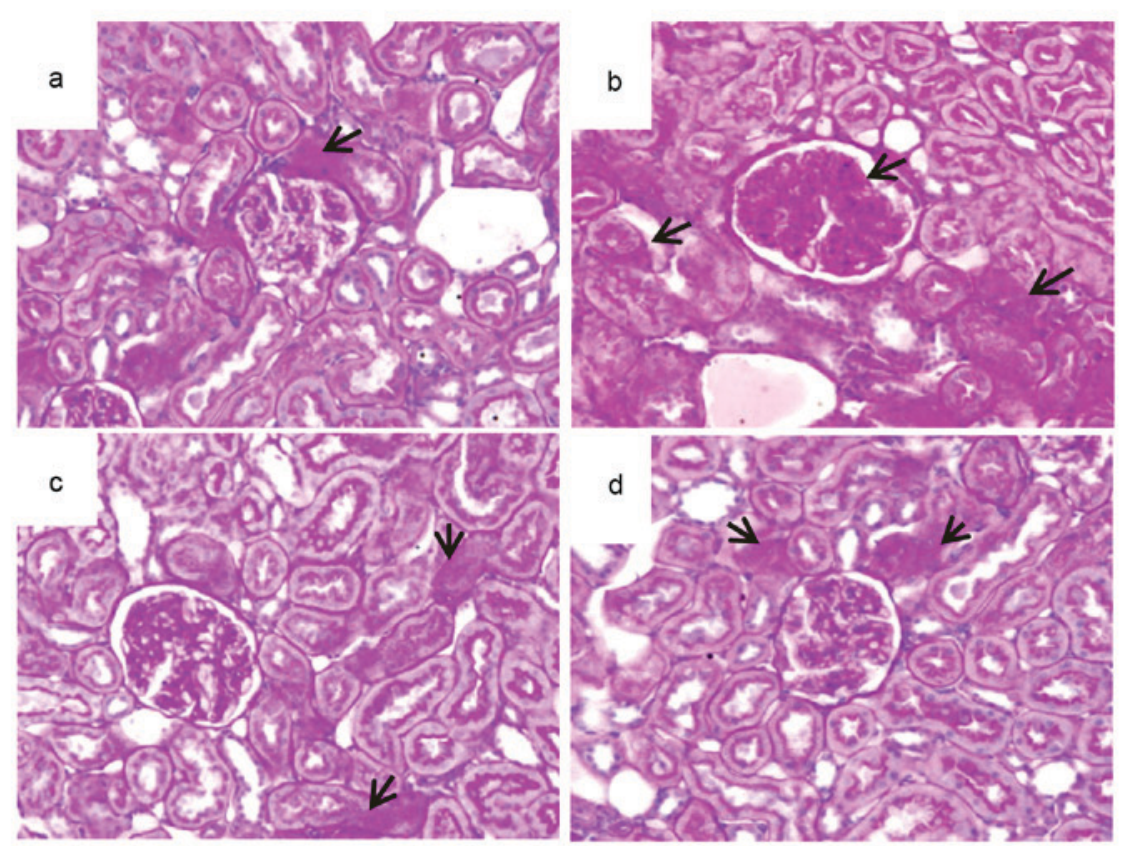

B
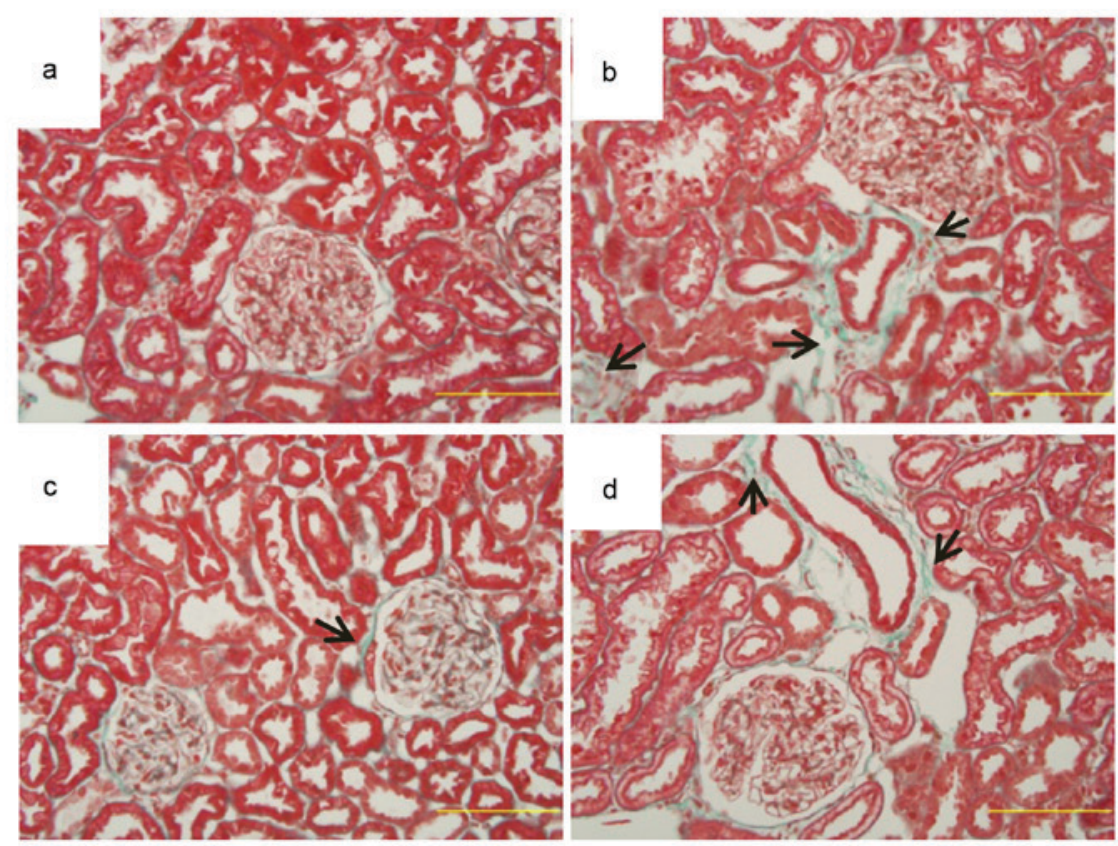

C
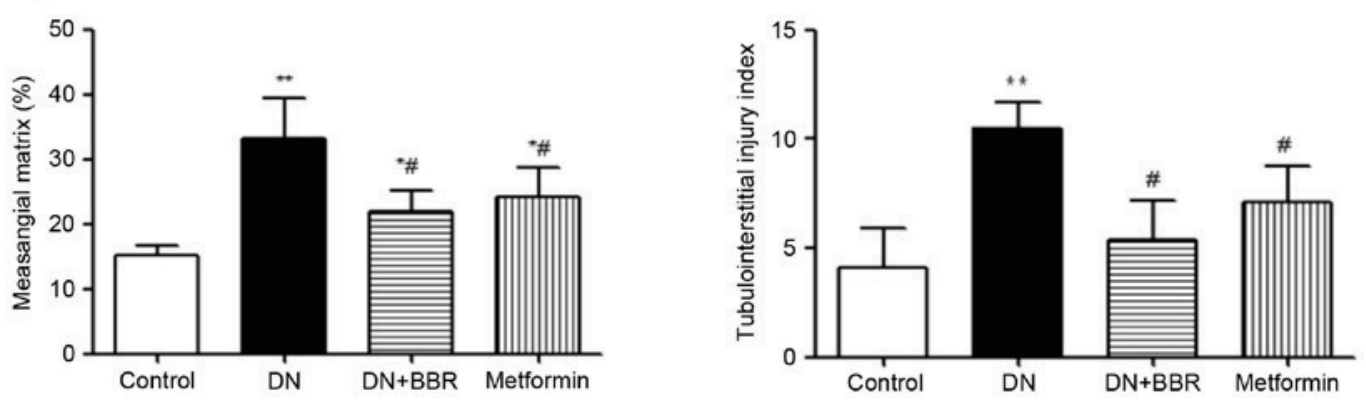

Figure 1. Staining of rat kidney tissues. (A) Periodic acid-Schiff staining of rat kidney tissues in the (A-a) control, (A-b) DN, (A-c) DN+BBR and (A-d) metformin groups. (B) Masson staining of the (B-a) control, (B-b) DN, (B-c) DN+BBR and (B-d) metformin groups. (C) Quantification of PAS and Masson staining. Data are presented as the mean \pm standard deviation. ${ }^{*} \mathrm{P}<0.05,{ }^{* *} \mathrm{P}<0.01$ vs. control group, ${ }^{\prime} \mathrm{P}<0.05$ vs. DN group. DN, diabetic nephropathy; BBR, berberine.

development of matrix production and the replacement of the normal renal tubulointerstitium with fibrous scarring; however, effective methods for alleviation of fibrosis during DN remain to be elucidated. 
A
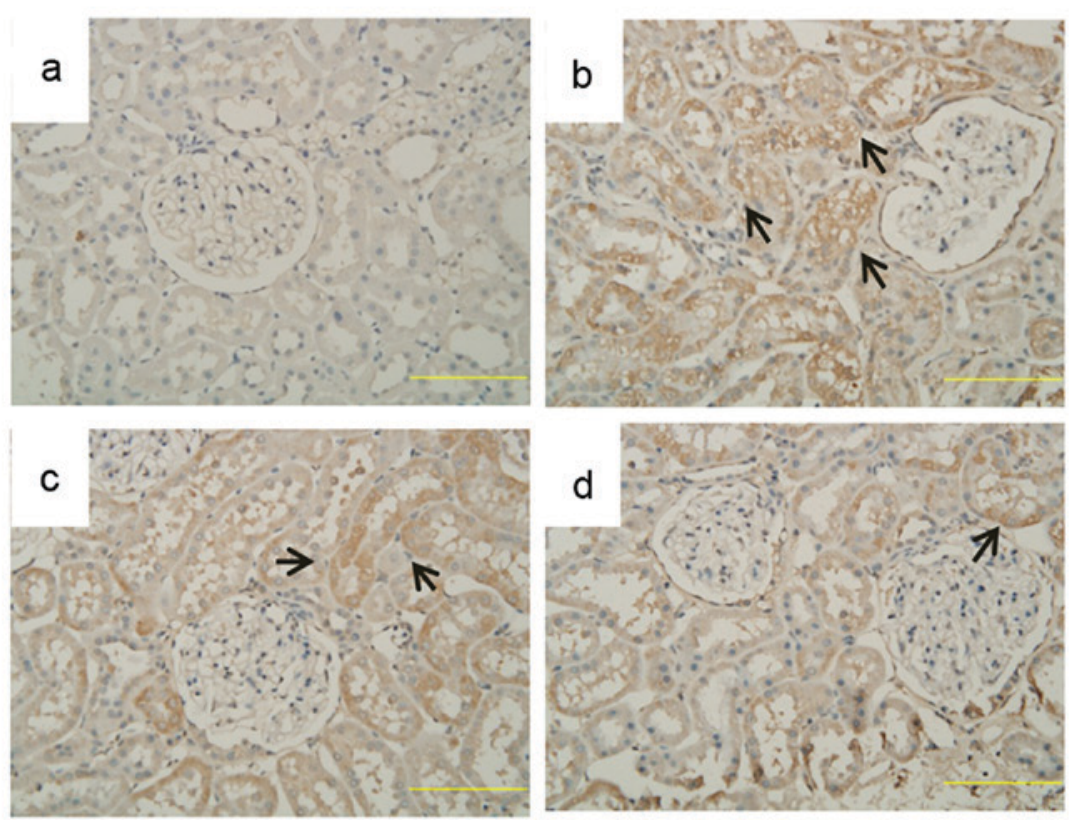

B
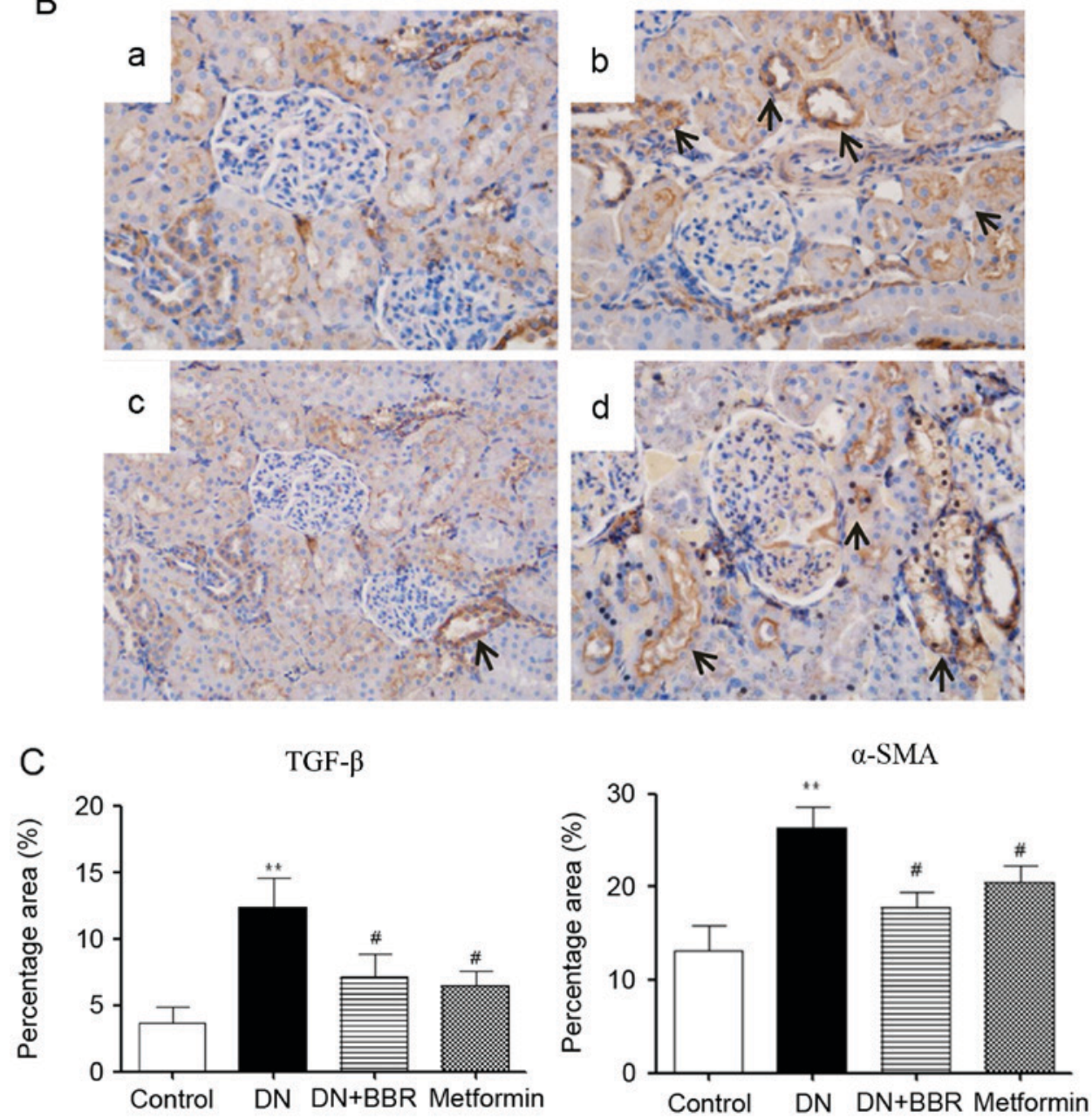

Figure 2. Immunohistochemical staining of rat kidneys. (A) TGF- $\beta$ expression in the (A-a) control, (A-b) DN, (A-c) DN+BBR and (A-d) metformin groups (B) $\alpha$-SMA expression in the (B-a) control, (B-b) DN, (B-c) DN+BBR and (B-d) metformin groups. (C) Quantification of TGF- $\beta$ and $\alpha$-SMA staining. Data are presented as the mean \pm standard deviation. ${ }^{* *} \mathrm{P}<0.01$ vs. control group, ${ }^{*} \mathrm{P}<0.05$ vs. DN group. TGF- $\beta$, transforming growth factor- $\beta ; \alpha$-SMA, $\alpha$ smooth muscle actin; DN, diabetic nephropathy; BBR, berberine.

High fat food combined with STZ injection may exacerbate kidney injury. Previous studies determined that seriously injured rat kidneys also exhibit increased levels of Scr 12 weeks after induction of the diabetic model $(16,17)$. 
A

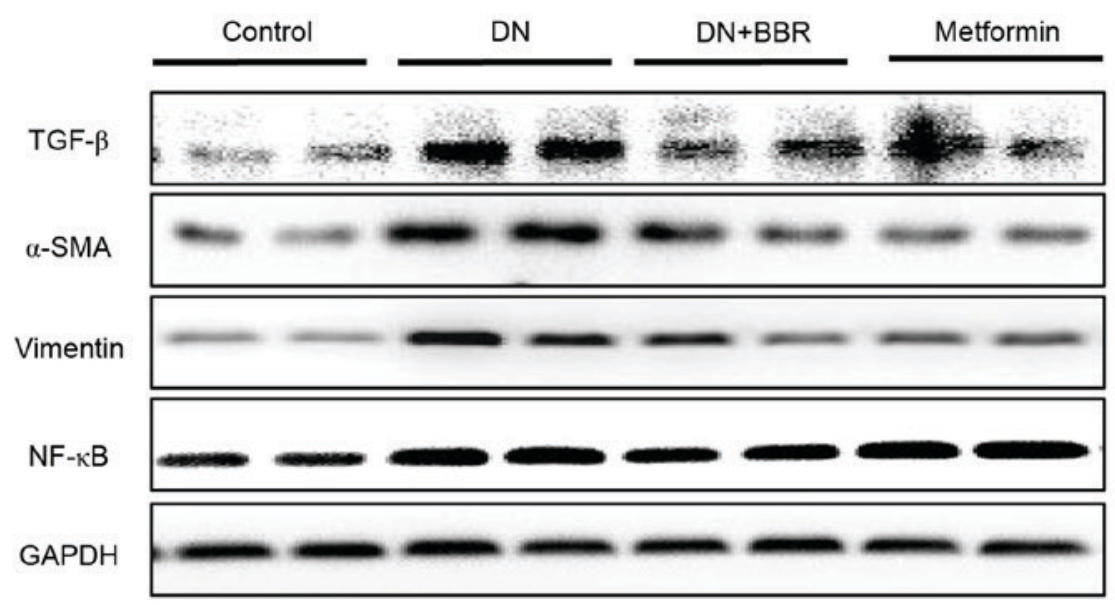

B
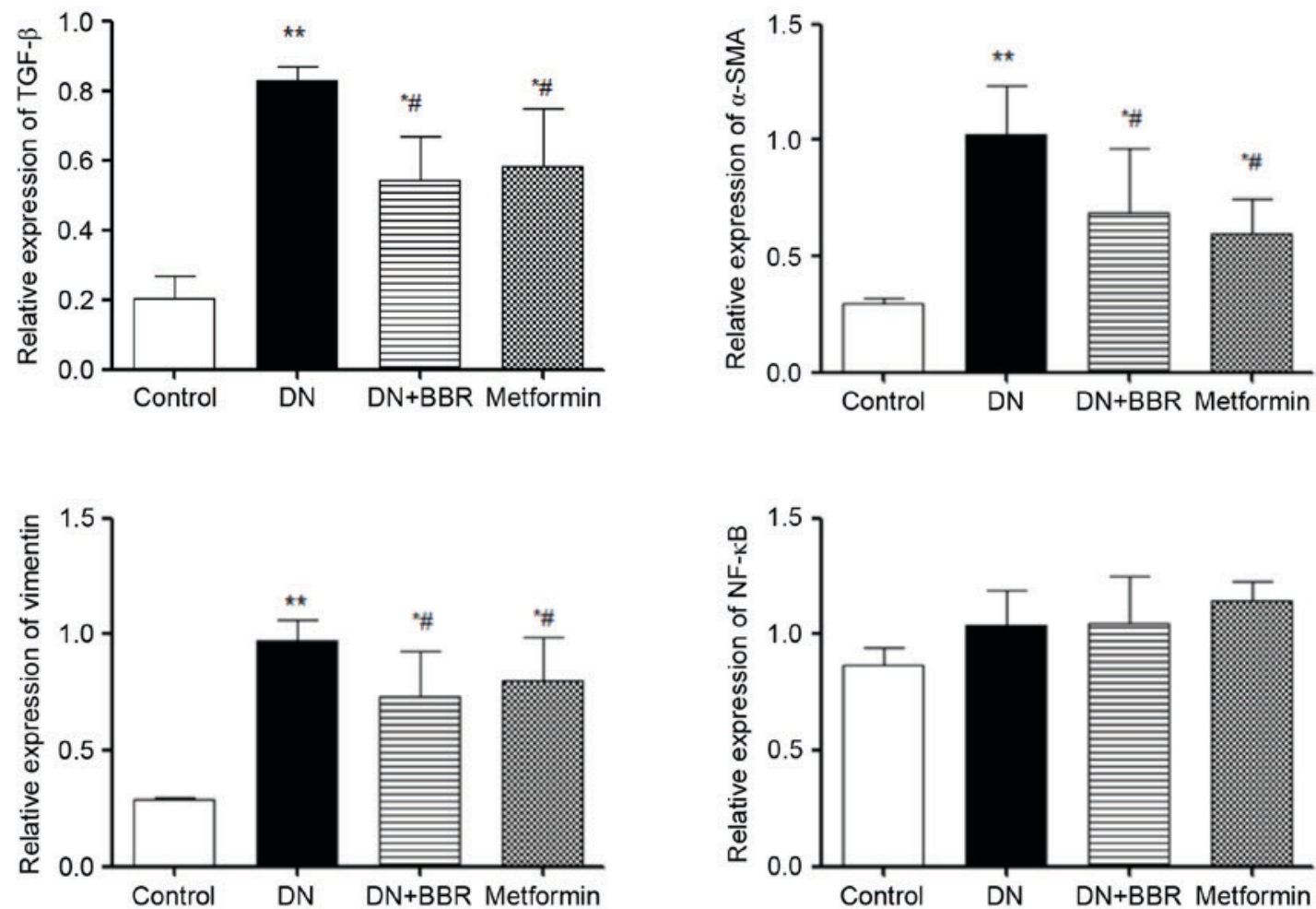

C

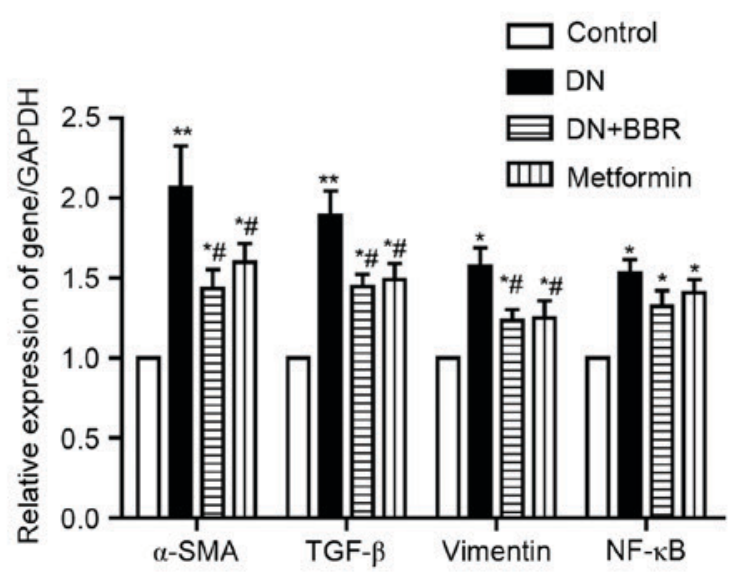

Figure 3. Protein and mRNA expression levels in rat kidneys. (A) Representative western blot images and (B) quantification of protein expression levels of TGF- $\beta, \alpha$-SMA, vimentin and NF- $\mathrm{B}$. (C) mRNA expression levels were determined using reverse transcription-quantitative polymerase chain reaction. GAPDH served as an internal control. Data are presented as the mean \pm standard deviation. ${ }^{*} \mathrm{P}<0.05$ and ${ }^{* *} \mathrm{P}<0.01$ vs. control group, ${ }^{\#} \mathrm{P}<0.05$ and ${ }^{\# \#} \mathrm{P}<0.01$ vs. DN group. TGF- $\beta$, transforming growth factor- $\beta$; $\alpha$-SMA, $\alpha$ smooth muscle actin; NF- $\kappa$, nuclear factor $\kappa \mathrm{B}$; DN, diabetic nephropathy; BBR, berberine. 
The present study determined that BBR effectively reduced the serum Scr and BUN levels, which was in accordance with previous studies $(18,19)$. In addition, BBR treatment also reduced the expression of TGF- $\beta$ and $\alpha$-SMA, which have been identified to have an important role in the ECM-synthesizing process. These findings have demonstrated that BBR may have a significant protective effect against diabetic renal injury.

The earliest detectable alteration in the pathogenesis of diabetic nephropathy is an expansion of the glomerular mesangium, which occurs due to excessive accumulation of ECM proteins. During this process, TGF- $\beta$ and $\alpha$-SMA have been identified to have an important role in the ECM. TGF- $\beta$ contributes to a variety of biological processes, including cell proliferation, differentiation, apoptosis, autophagy and production of the ECM. A previous study revealed that TGF- $\beta$ was upregulated in injured kidneys in both patients and animal disease models (20). The downregulation of TGF- $\beta$ signaling pathways may alleviate kidney fibrosis $(21,22)$. During DN, fibroblasts are a critical component of the repair mechanism, and increased TGF- $\beta$ may lead to an increased transformation of these cells into activated myofibroblasts, indicated by increased expression of $\alpha$-SMA. Hinz et al (23) reported that increased $\alpha$-SMA expression enhances fibroblast contractile activity (23). Recent studies have identified a limited number of drugs that may reverse fibrosis $(24,25)$. However, in present study, Chinese herbs have been identified to have a favorable curative effect in fibrosis.

Traditional Chinese herbs have been widely used in the treatment of diabetes in China for thousands of years. Currently, various nutraceutical ingredients, frequently of botanical origin, have been investigated in terms of their ability to treat fibrosis. Meng et al (26) determined that Astragali radix may alleviate renal tubulointerstitial fibrosis by inhibition of tubular EMT and fibroblast activation (26). $\mathrm{BBR}$, extracted from Coptis chinensis, was first proved to have anti-hyperglycemic activity in 1986 (27,28). However, its effect on DN, particularly in fibrosis, remains to be elucidated. The present study observed that BBR may inhibit the expression of TGF- $\beta$ and $\alpha$-SMA in kidney tissues. This may contribute to its ability to ameliorate the symptoms of DN.

In conclusion, the present study demonstrated that BBR, extracted from Rhizoma coptidis, had a beneficial effect on TGF- $\beta$ and $\alpha$-SMA expression levels. Furthermore, BBR treatment improved renal fibrosis in DN. However, BBR was not observed to regulate blood glucose in the present study. Future studies should focus on the underlying molecular mechanism in order to allow for the clinical use of BBR.

\section{Acknowledgements}

The current study was supported by a grant from the Henan Province Science and Technology Key Project (grant no. 162102310256).

\section{References}

1. Atkins RC and Zimmet P: Diabetic kidney disease: Act now or pay later. Kidney Int 77: 375-377, 2010.

2. Cooper ME: Diabetes: Treating diabetic nephropathy-still an unresolved issue. Nat Rev Endocrinol 8: 515-516, 2012.
3. Reidy K, Kang HM, Hostetter T and Susztak K: Molecular mechanisms of diabetic kidney disease. J Clin Invest 124: 2333-2340, 2014.

4. Badal SS and Danesh FR: New insights into molecular mechanisms of diabetic kidney disease. Am J Kidney Dis 63 (2 Suppl 2): S63-S83, 2014.

5. Fan Y, Li X, Xiao W, Fu J, Harris RC, Lindenmeyer M, Cohen CD, Guillot N, Baron MH, Wang N, et al: BAMBI elimination enhances alternative TGF- $\beta$ signaling and glomerular dysfunction in diabetic mice. Diabetes 64: 2220-2233, 2015.

6. Du J, Hong S, Dong L, Cheng B, Lin L, Zhao B, Chen YG and Chen $X$ : Dynamic sialylation in transforming growth factor- $\beta$ (TGF- $\beta$ )-induced epithelial to mesenchymal transition. J Biol Chem 290: 12000-12013, 2015.

7. Chen KH, Hung CC, Hsu HH, Jing YH, Yang CW and Chen JK: Resveratrol ameliorates early diabetic nephropathy associated with suppression of augmented TGF- $\beta /$ smad and ERK1/2 signaling in streptozotocin-induced diabetic rats. Chem Biol Interact 190: 45-53, 2011.

8. Chen X, Zhang Y,Zhu Z, Liu H, Guo H, Xiong C, Xie K, Zhang X and Su S: Protective effect of berberine on doxorubicin-induced acute hepatorenal toxicity in rats. Mol Med Rep 13: 3953-3960, 2016.

9. Adil M, Kandhare AD, Dalvi G, Ghosh P, Venkata S, Raygude KS and Bodhankar SL: Ameliorative effect of berberine against gentamicin-induced nephrotoxicity in rats via attenuation of oxidative stress, inflammation, apoptosis and mitochondrial dysfunction. Ren Fail 38: 996-1006, 2016.

10. Liu C, Wang Z, Song Y, Wu D, Zheng X, Li P, Jin J, Xu N and Li L: Effects of berberine on amelioration of hyperglycemia and oxidative stress in high glucose and high fat diet-induced diabetic hamsters in vivo. Biomed Res Int 2015: 313808, 2015.

11. Zhang H, Li P, Burczynski FJ, Gong Y, Choy P, Sha H and Li J: Attenuation of diabetic nephropathy in otsuka long-evans tokushima fatty (OLETF) rats with a combination of Chinese herbs (Tangshen Formula). Evid Based Complement Alternat Med 2011: 613737, 2011.

12. Zhao TT, Zhang HJ, Lu XG, Huang XR, Zhang WK, Wang H, Lan HY and Li P: Chaihuang-Yishen granule inhibits diabetic kidney disease in rats through blocking TGF- $\beta / \mathrm{Smad} 3$ signaling. PLoS One 9: e90807, 2014.

13. Hou CC, Wang W, Huang XR, Fu P, Chen TH, Sheikh-Hamad D and Lan HY: Ultrasound-microbubble-mediated gene transfer of inducible Smad7 blocks transforming growth factor-beta signaling and fibrosis in rat remnant kidney. Am J Pathol 166: 761-771, 2005

14. Livak KJ and Schmittgen TD: Analysis of relative gene expression data using real-time quantitative PCR and the 2(-Delta Delta C(T)) method. Methods 25: 402-408, 2001.

15. Liu SF, Chang SY, Lee TC, Chuang LY, Guh JY, Hung CY, Hung TJ, Hung YJ, Chen PY, Hsieh PF and Yang YL: Dioscorea alata attenuates renal interstitial cellular fibrosis by regulating Smad- and epithelial-mesenchymal transition signaling pathways. PLoS One 7: e47482, 2012.

16. Renno WM, Abdeen S, Alkhalaf M and Asfar S: Effect of green tea on kidney tubules of diabetic rats. Br J Nutr 100: 652-659, 2008.

17. Kuo CW, Shen CJ, Tung YT, Chen HL, Chen YH, Chang WH, Cheng KC, Yang SH and Chen CM: Extracellular superoxide dismutase ameliorates streptozotocin-induced rat diabetic nephropathy via inhibiting the ROS/ERK1/2 signaling. Life Sci 135: 77-86, 2015.

18. Wang J, Liu H, Li N, Zhang Q and Zhang H: The protective effect of fucoidan in rats with streptozotocin-induced diabetic nephropathy. Mar Drugs 12: 3292-3306, 2014.

19. Mapanga RF, Tufts MA, Shode FO and Musabayane CT: Renal effects of plant-derived oleanolic acid in streptozotocin-induced diabetic rats. Ren Fail 31: 481-491, 2009.

20. Rodell CB, Rai R, Faubel S, Burdick JA and Soranno DE: Local immunotherapy via delivery of interleukin-10 and transforming growth factor $\beta$ antagonist for treatment of chronic kidney disease. J Control Release 206: 131-139, 2015.

21. Yang Y, Kim B, Park YK, Koo SI and Lee JY: Astaxanthin prevents TGF $\beta 1$-induced pro-fibrogenic gene expression by inhibiting Smad3 activation in hepatic stellate cells. Biochim Biophys Acta 1850: 178-185, 2015.

22. Lu J, Shi J, Li M, Gui B, Fu R, Yao G, Duan Z, Lv Z, Yang Y, Chen $Z$, et al: Activation of AMPK by metformin inhibits TGF- $\beta$-induced collagen production in mouse renal fibroblasts. Life Sci 127: 59-65, 2015. 
23. Hinz B, Celetta G, Tomasek JJ, Gabbiani G and Chaponnier C: Alpha-smooth muscle actin expression upregulates fibroblast contractile activity. Mol Biol Cell 12: 2730-2741, 2001.

24. Ramos AM, González-Guerrero C, Sanz A, Sanchez-Niño MD, Rodríguez-Osorio L, Martín-Cleary C, Fernández-Fernández B, Ruiz-Ortega M and Ortiz A: Designing drugs that combat kidney damage. Expert Opin Drug Discov 10: 541-556, 2015.

25. Meng XM, Tang PM, Li J and Lan HY: TGF- $\beta /$ Smad signaling in renal fibrosis. Front Physiol 6: 82, 2015.
26. Meng LQ, Tang JW, Wang Y, Zhao JR, Shang MY, Zhang M, Liu SY, Qu L, Cai SQ and Li XM: Astragaloside IV synergizes with ferulic acid to inhibit renal tubulointerstitial fibrosis in rats with obstructive nephropathy. Br J Pharmacol 162: 1805-1818, 2011.

27. Yao J, Kong W and Jiang J: Learning from berberine: Treating chronic diseases through multiple targets. Sci China Life Sci 58: 854-859, 2015.

28. Singh IP and Mahajan S: Berberine and its derivatives: A patent review (2009-2012). Expert Opin Ther Pat 23: 215-231, 2013. 